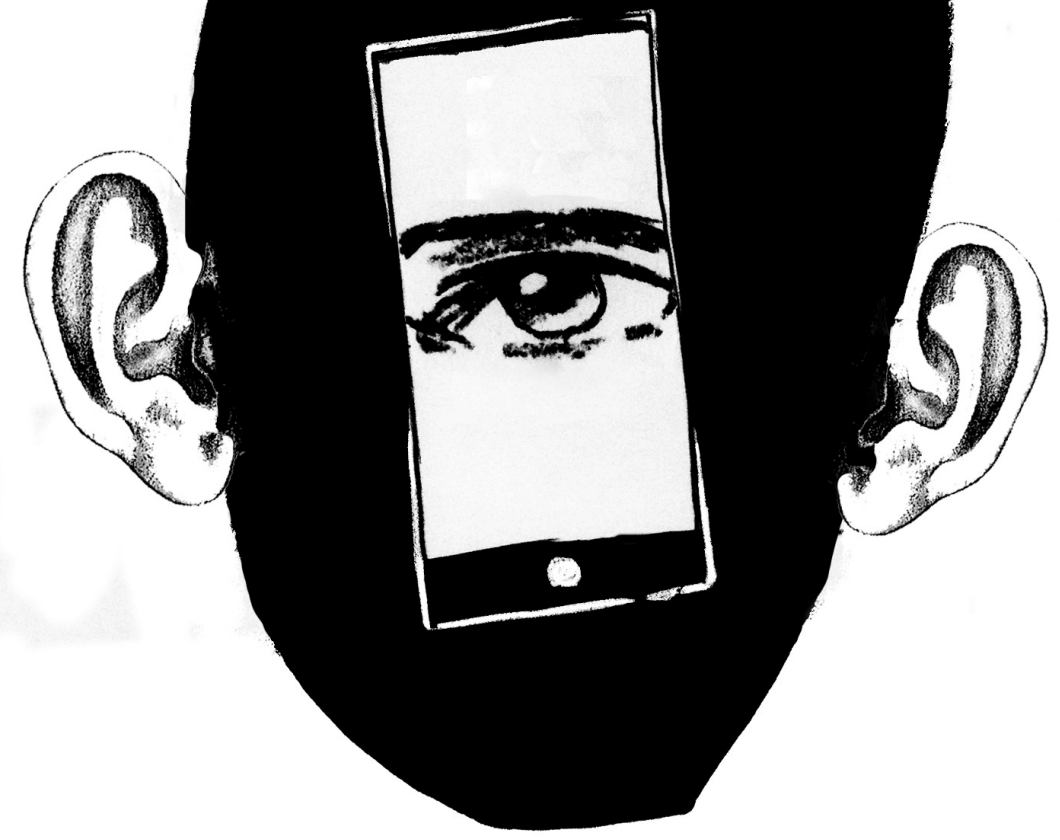

\title{
BREVE PANORAMA DE LA COMUNICACIÓN MÓVIL ACERCAMIENTO AL CASO COLOMBIANO
}

A BRIEF OVERVIEW OF MOBILE

COMMUNICATION. APPROACH TO THE

COLOMBIAN CASE.

Por

Ana María Díaz Jordán ${ }^{1}$

Universidad del Valle

ana.diaz.jordan@correounivalle.edu.co

Andrés Felipe Castañeda ${ }^{2}$

Universidad Santiago de Cali

andres.castaneda00@usc.edu.co 
Resumen: Este documento tiene como propósito aportar al análisis de las implicaciones socioculturales de la incorporación de los celulares en la sociedad actual, para el caso específico, de Colombia, y así comprender los cambios en las lógicas comunicativas que han dado pie a una cibercultura.

Para esto construiremos un breve panorama de la comunicación móvil en Colombia: Iniciaremos con un paneo por diferentes términos que permiten sustentar a nivel teórico la propuesta. Posteriormente, continuaremos con la exploración de algunos textos en América latina para finalizar en Colombia, con una revisión sobre la penetración de los celulares como artículos indispensables en la cotidianidad.

Palabras Clave: Cibercultura, Comunicación móvil, Usos socioculturales.

Abstract: This document aims to contribute to the analysis of the sociocultural implications of the incorporation of cellphones in today's society, for the specific case, of Colombia, and thus understand the changes in the communicative logics that have given rise to a cyberculture.

For this we will build a brief overview of mobile communication in Colombia. We will start with a panning for different terms that allow to support the proposal at a theoretical level. Subsequently, we will continue with the exploration of some texts that in Latin America and, specifically, in Colombia, present a review on the penetration of cell phones as indispensable articles in daily life.

Keywords: Cyberculture, Mobile communication, Socio-cultural uses.

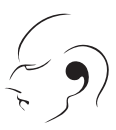

\section{El uso del celular en Colombia, un campo poco explorado}

En Colombia el teléfono inteligente es considerado un artículo de uso personal y compañía necesaria para los usuarios. La mayoría de los consumidores usan su teléfono inteligente en cada oportunidad que se les presenta. De la muestra de 943 casos, seleccionada para la encuesta de Consumidores Móviles 2018 de la firma Deloitte, el 56\% consulta su teléfono celular mientras ve una película, el 45\% mientras va en el transporte público, el $27 \%$ mientras come y un 22\% mientras camina o cruza una calle. La ansiedad por estar conectados lleva a que un $50 \%$ de los usuarios, aunque no reciban una notificación, revise continuamente su teléfono celular. 
El mercado de los teléfonos inteligentes, además de ser sobresaliente en cifras de venta, cuenta con una alta tasa de innovación y cambio -obsolescencia programada ${ }^{3}$ en comparación con otros dispositivos electrónicos de consumo masivo (Deloitte, 2018). Hace algunos años los móviles fueron considerados elemento característico de los jóvenes entre 18 y 24 años; hoy el uso de estos dispositivos se ha popularizado entre los adultos de 35 a 45 años (Deloitte, 2018). Las aplicaciones líderes se siguen relacionando principalmente con la necesidad de comunicarnos y de interactuar con otros, por ejemplo, la mensajería instantánea es la aplicación más utilizada por los propietarios de teléfonos inteligentes y la actividad que se realiza con mayor frecuencia es la de mirar videos cortos -a través de Youtube- seguida de los videojuegos y el consumo de contenidos: videos, música y noticias (Deloitte, 2018).

Más allá de los números y porcentajes, es necesario realizar una interpretación: tratar de trascender el dato empírico para entender y reconocer cómo estos medios se han convertido en una extensión de los sentidos humanos. Toda esta elaboración nos conduce a poner de manifiesto el propósito principal del presente documento: aportar en la identificación de algunas de las implicaciones socioculturales del uso de la comunicación móvil, presentando un breve panorama sobre el consumo de este medio a nivel mundial y algunas particularidades en lo local.

\title{
¿Teléfono inteligente para un usuario inteligente?
}

\author{
"Nos acercamos al final de 'la década de Internet' y el móvil se sitúa en el \\ centro de nuestra conectividad, es el dispositivo con el que nos despertamos y nos \\ acostamos cada día. El móvil, en plena era del 'infotainment' se ha convertido en \\ "la pantalla que nunca se apaga",
}

(Fumero, 2010).

La primera década de este siglo será recordada en los estudios de comunicación como una época de transición entre la dominancia de los medios de comunicación de masas y el surgimiento de diferentes formas de consumo individual de información o entretenimiento. Las implicaciones de este nuevo orden impactan profundamente los procesos de consumo cultural, los agentes y el contexto en el que se desarrolla esta actividad. Nuevos modelos de empresa, convergencia en cuanto a habilidades profesionales y objetos de estudio, perfiles difusos ¿quién produce y quién consume?, innovadoras técnicas de creación y distribución de contenidos hacen parte de esta convulsa metamorfosis.

La movilidad abarca una variedad de dispositivos portátiles e inalámbricos como teléfonos inteligentes, tabletas, videoconsolas portátiles, PC ultramóvil y mecanismos electrónicos de lectura o e-readers, que más allá de su alta complejidad técnica, se han logrado imponer gracias a una "elevada tasa de penetración y [...] conectividad a la red” (Costa-Sánchez, 2014, p.676). Estos dispositivos proponen escenarios inéditos en el consumo: no es sólo la manera en cómo se accede a la información, es también la interpretación y la mediación. 
Los dispositivos móviles representan en la actualidad un paradigma de la concepción macLuhaniana a partir de la cual una tecnología se concibe como una extensión del cuerpo humano, de nosotros mismos y como extensión de los sentidos que "afecta a todo el complejo psíquico y social y ejerce un efecto insospechado en nuestras vidas" (García, 2014, p. 275).

Entre la variedad de dispositivos mencionados, tomaremos como referente al teléfono móvil, conocido tradicionalmente como celular, que nace del desarrollo de las telecomunicaciones y el teléfono, pero que debido a la convergencia de plataformas y funcionalidades hoy logra fusionar multimedia y navegación en red, convirtiéndolo en un dispositivo inteligente. Por detrás de los históricos medios tradicionales como el cine, la televisión e Internet, esta "cuarta pantalla" ofrece un gran potencial en el ámbito de la personalización y la movilidad (Ahonen, 2008), al diluir las fronteras entre lo público y lo privado, afectando la concepción del espacio-tiempo.

De acuerdo con la encuesta global de consumidores móviles 2018, versión Colombia (Deloitte), las tendencias en ventas indican que año a año se reduce la demanda por compra de computadores de escritorio (34\%). Este dato contrasta con el aumento en la venta de dispositivos inteligentes (96\%), computadores portátiles (47\%) y tabletas (38\%).

Las dinámicas de las industrias culturales, en modo general, y de los medios de comunicación de manera particular, han sido modificadas por los dispositivos móviles inteligentes, proponen nuevas formas de contar historias, renuevan modalidades expresivas y complejizan los sistemas de interacción comunicativa; también, se conciben cambios radicales "en las formas de producción y distribución de contenidos, con estrategias para la realización multiplataforma y para el aprovechamiento del potencial de los usuarios no sólo como fuente, sino también como generadores de mensajes" (García, Westlund \& Silva, 2015, p.133).

\section{El celular: herramienta, lujo y necesidad}

Continuaremos, entonces, con un paneo por diferentes términos que permiten sustentar a nivel teórico este documento. Posteriormente, haremos la exploración de algunos textos que en América latina y, en específico, a nivel de Colombia, presentan una revisión sobre la penetración de los celulares como artículos indispensables en la cotidianidad.

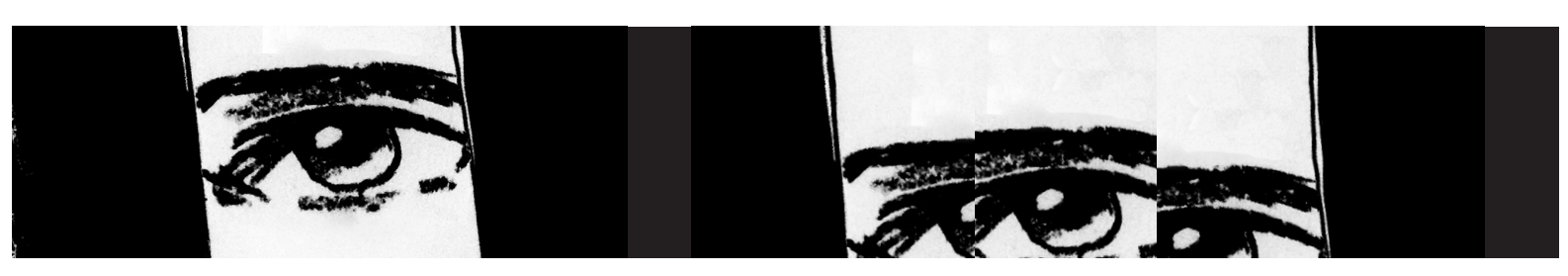




\section{De la sociedad del industrialismo a la sociedad red}

De acuerdo con Armand Mattelard (2007), la idea de una sociedad de la información es anterior a la aparición de esta noción en el lenguaje y la cultura de la modernidad. Desde el siglo XVII se avizoraba un interés inspirado en la mística del número y se proponía un panorama que contemplaba la perfección de la sociedad basada en la razón. Para finales del siglo XVIII e inicios del XIX, se observa un cambio de actitud respecto a la concepción del tiempo y el espacio que impacta no sólo las operaciones de ultramar, el cálculo de probabilidades o la difusión de datos dirigidos a negociantes y financieros, sino también a la cotidianidad. Poco a poco se empieza a construir una nueva forma de pensar, un ideal de lograr proponer un lenguaje analítico que permitiría el conocimiento de la verdad y haría casi imposible la existencia del error, un proyecto de conocimiento y racionalidad universal que involucra a la razón y también a las palabras.

Durante la era industrial, la estadística se convierte en la ciencia reina y "el hombremedio" se transforma en el centro de gravedad. El poder predecir lo que va a suceder a través de los números da la posibilidad de entender a la sociedad como una gran industria que se puede moldear, organizar y asegurar. Es a este modelo de organización al que Castells (2006) se refiere como la sociedad del industrialismo sistémico, en la que las tecnologías se agrupan "alrededor del núcleo de la energía (...) convergiendo en varios campos, de la ingeniería química y la metalurgia, al transporte, las telecomunicaciones y, recientemente, a las ciencias de la vida y sus aplicaciones" (Castells, 2006, p. 33).

Poco a poco, se continúa configurando la idea de generar simbiosis con un pensamiento en red, universal, técnico y a la vez social. La conformación de bibliotecas y museos internacionales de métodos documentales, así como la existencia de instituciones políticas con el propósito de "hacer del mundo entero una sola ciudad y de todos los pueblos una sola familia" (Mattelart, 2007, p. 52), son muestra de ello. El surgimiento de los medios de comunicación masiva acelera los flujos de información y posibilita la aparición de los públicos modernos. "Contrariamente a lo que la fascinación por la sociedad de redes podrá hacer creer un siglo más tarde, la representación reticular del planeta es, pues, muy anterior a lo que se ha convenido en llamar "revolución de la información" (Mattelart, 2007, p. 53).

Entonces, si la idea de una sociedad conectada a través de los diversos nodos de una red se ha venido configurando desde tiempo atrás ¿cuál es la gran diferencia que supone el momento actual? ¿De dónde surge el interés por denominar este punto de la historia "sociedad del conocimiento", "era de la información" o "sociedad red"?

Usar el término sociedad del conocimiento para identificar específicamente a una época es un error intelectual (Castells, 2006), por la sencilla razón de que "todas las sociedades conocidas se han basado en la información y el conocimiento como 
fuentes de poder, riqueza y significado" (Mokyr, 1990; Mazlish, 1993). Un término más ajustado sería el de sociedad informacional, que como paradigma tecnológico compone la base de las sociedades del siglo XXI y que se caracteriza por "el aumento de la capacidad de procesamiento de la información y la comunicación humana, hecho posible por la revolución de la microelectrónica, el software y la ingeniería genética" (Castells, 2006, p. 34).

La expansión y el crecimiento del cuerpo humano, gracias a la proliferación de equipos portátiles, permiten la comunicación ubicua, lo que posibilita a las personas interactuar "en cualquier momento, desde cualquier lugar, confiando en una infraestructura de apoyo que administra recursos materiales distribuidos a lo largo y ancho de una parrilla electrónica de información” (Castells, 2006, p. 31). Esto afecta la forma como la mente humana procesa información. Por tanto, la gran revolución de nuestro tiempo radica en las formas particulares que adopta el flujo de la información y de la comunicación.

Sobre esta base se expande una nueva estructura social que construye los cimientos de una sociedad red, cuya fuerza está dada por la flexibilidad, adaptabilidad y capacidad de autoreconfiguración. "La tecnología digital electrónica permite un aumento de procesamiento de información sin precedentes históricos, no sólo en cuanto a volumen, sino también en la complejidad de las operaciones y en la velocidad de procesamiento de la comunicación" (Castells, 2006, p. 35). El reto que se impone es conectar lo global con lo local, el espacio de los flujos de información con los lugares que habitan las personas. Es en este trasegar en el que surge internet como tecnología digital que permite a los seres humanos superar limitantes de tiempo y espacio.

\section{Del hipertexto al internet de las cosas}

Durante sus años de existencia, internet ha tenido múltiples cambios y transformaciones. Un primer momento se caracteriza por la circulación de hipertextos a través de una red informática global. Esta red, a la que se le denominó Arpanet, surge en 1983 como una opción a un sistema de almacenamiento y circulación de datos de forma simultánea, pero sin un nodo o punto fijo en términos de espacialidad. Un protocolo del sistema de defensa de los Estados Unidos para evitar ataques y destrucción de su información.

Arpanet salió del espacio militar para insertarse en la universidad y desde allí se abrió al mundo con el nombre de internet. Las páginas web de este primer ciclo, que se diseñaban en el ordenador y posteriormente se subían a la red, eran estáticas, similares a las fotografías. El proceso de retroalimentación se daba a través del correo electrónico, era un ser humano el que mediaba toda la relación entre el medio y los

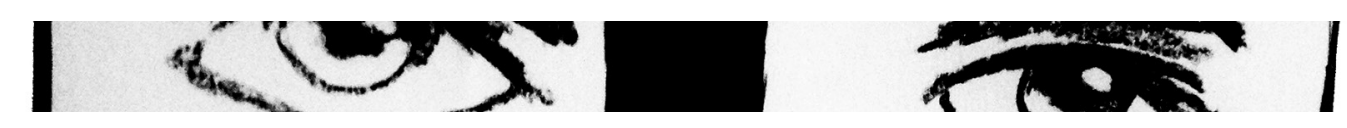


usuarios. Este primer internet adoptó el modelo de los medios de comunicación tradicionales, en donde se mantenía la disposición a la producción de uno para muchos: "una fuente productora para una audiencia receptora o consumidora" (Sánchez, Botero \& Giraldo, 2012, p. 72).

Un segundo momento es conocido como internet 2.0; en él se favorece eminentemente la comunicación hombre-máquina gracias a los procesos de automatización. Es a lo que Norbert Winner se refirió en su teoría cibernética de la comunicación como la necesidad de combatir la entropía: "La información, las máquinas que la tratan y las redes que estas tejen son las únicas capaces de luchar contra la tendencia a la entropía" (Citado por Mattelart \& Mattelart, 2001, p. 47). La sociedad de la información sólo existe si hay un intercambio en el que los datos puedan circular.

Internet 2.0 permite un flujo de información sin la mediación necesaria del ser humano: bases de datos generan algoritmos, robots programan y permiten crear formularios que luego son diligenciados por los seres humanos, verificados por la máquina y enviados a una base de datos que posteriormente remitirá de forma automática un correo de confirmación. El modelo del internet dinámico implica que "muchos producen para muchos a partir de la producción de muchos. Podría decirse que todos producen para todos, a partir de la producción de todos" (Sánchez, Botero, Giraldo, 2012, p. 72). El usuario deja de ser pasivo y se convierte en un productor que interactúa de forma cibernética con máquinas. De ahí el término prosumer, consumidor y generador de contenidos.

Otra característica de este segundo momento es el uso de plataformas basadas en la colaboración como los chats, las comunidades, los foros, las wikis y otras posibilidades de acceso y construcción colectiva. Con la web 2.0 se popularizan términos como crowdsourcing: multitudes que buscan y construyen información.

El tercer momento, conocido como la web semántica "propicia altísimos procesos de personalización en la interacción con los contenidos" (García, 2014, p. 273), mediante técnicas de georeferenciación y geolocalización. Respecto a la primera etapa donde una dificultad era conocer con claridad el perfil de los usuarios, la web semántica se vale de la parametrización para proporcionar datos en detalle de quien navega. Se pasa de una comunicación en masa con producción centralizada de contenidos, a una comunicación personalizada en masa, lo que permite a un sitio web proponer diferentes interfaces para sus usuarios de acuerdo a la información que de estos se tenga. Ejemplo de lo anterior es la configuración de experiencias de usuario de acuerdo a búsquedas anteriores o al país donde se encuentre.

Hoy asistimos al internet de las cosas, que ha sido definido desde el Instituto de Tecnología de Massachusetts (MIT) como el punto en el tiempo en el que se conectaron a Internet más “cosas u objetos” que personas. 
En 2003, había aproximadamente 6,3 mil millones de personas en el planeta, y había 500 millones de dispositivos conectados a Internet. Si dividimos la cantidad de dispositivos conectados por la población mundial, el resultado indica que había menos de un dispositivo $(0,08)$ por persona. (...) El crecimiento explosivo de los smartphones y las tablet elevó a 12,5 mil millones en 2010 la cantidad de dispositivos conectados a Internet, en tanto que la población mundial aumentó a 6,8 mil millones, por lo que el número de dispositivos conectados por persona es superior a 1 (1,84 para ser exactos) por primera vez en la historia. (Evans, 2011).

El internet de las cosas está compuesto por una colección dispersa de redes que tienen distintos fines y que generan gran cantidad de datos - Big Data- disponibles para ser procesados, identificar tendencias, patrones y generar conocimiento. Este es un internet que sale del pc y se instaura en lo cotidiano ${ }^{5}$. El internet de las cosas es deslocalizado y ha colonizado fuertemente otras pantallas como las del smarthphone, que se convierte en una extensión del cuerpo humano: asistimos a la creación del hombre-máquina en donde más de la mitad de la población en línea afirma que se conecta a Internet a través de sus dispositivos móviles (Google Consumer Barometer, 2017) Estos teléfonos cada vez utilizan formas más sofisticadas de identificación y autenticación del usuario dueño del dispositivo para garantizar su seguridad códigos, huellas e iris ${ }^{6}$ - pero también, para dar respuesta a solicitudes y necesidades por medio de funciones de aprendizaje automático a través de la recolección de información relacionada con características y comportamientos personales ${ }^{7}$.

\section{La evolución de la telefonía móvil: de la voz al multimedia}

El teléfono celular que conocimos como un artefacto para realizar y recibir llamadas a distancia de forma inalámbrica ya no existe. Ahora los usuarios llevan consigo pequeñas computadoras de bolsillo conectadas las 24 horas del día a la internet y que combinan entre sus funciones aplicaciones (pequeños softwares) que suplen servicios de correo electrónico, ubicación geoespacial, mensajería instantánea, soportes multimedia, almacenamiento de archivos y un abanico de funciones a las que habría que dedicar un documento aparte.

Así como con la red se pueden identificar diferentes momentos en su evolución, la producción y diseño de celulares se divide en generaciones marcadas por los adelantos tecnológicos y ventajas que cada una ofrece. En 1979 la primera generación (1G) apareció y se enfocó en unas condiciones analógicas y exclusivamente su tecnología se dedicó a la transmisión de voz. Diez años más tarde apareció la Segunda generación (2G), esta nueva versión hizo una transición de lo analógico a lo digital. Los nuevos sistemas transmitían la voz a mayor velocidad, pero los datos móviles no tenían esas mismas ventajas. También se incorporaron servicios como datos, fax y mensajería de texto. La tercera generación (3G) arribó en 2001 con las altas velocidades de los protocolos de transmisión de información e incorporó aplicaciones para el manejo del audio en formato mp3, video, acceso rápido a Internet, voz y telecomunicaciones 
con video-conferencia. En conclusión, fue la generación ideal de las aplicaciones multimedia y gran tráfico de datos (Treviño y Millán, 2007). La cuarta generación (4G) surgió en febrero de 2007, cuando la firma japonesa NTT DoCoMo hizo el lanzamiento de un prototipo que buscaba que las velocidades máximas de transmisión de datos estuvieran entre $100 \mathrm{Mbit} / \mathrm{s}$ (12,5 MB/s) para una movilidad alta y $1 \mathrm{Gbit} / \mathrm{s}(125 \mathrm{MB} / \mathrm{s})$ para movilidad baja; esto respecto a datos móviles, pues la conectividad WiFi tendría mejor rendimiento. En conclusión, esta generación enfocó sus esfuerzos en el rendimiento de la conectividad que contribuyó a mejores condiciones de desarrollo de las actividades multimedia, en especial el video ${ }^{8}$. La próxima generación $(5 \mathrm{G})$ ha empezado a aparecer en el mercado desde finales del 2018 y se espera que para el año 2020 consolide su expansión por el mundo. Más allá de las mejoras en la velocidad, se espera que esta tecnología por fin le brinde el empujón necesario al ecosistema del Internet de las Cosas en el que las redes pueden satisfacer las necesidades de comunicación de miles de millones de dispositivos conectados a la internet, con un equilibrio justo entre velocidad, latencia y costo (Gemalto, 2019).

Las transformaciones que tanto la internet como los teléfonos móviles han tenido no pueden ser entendidas como mera técnica, es necesario analizar las implicaciones socioculturales de estos cambios, para así intentar comprender las lógicas comunicativas que han dado pie a una cibercultura. Este término se refiere a una colección de culturas y productos culturales que existen en y/o se hacen realizables a través de internet (Salinas \& Thompson, 2011). Considerar a la cibercultura como un sistema dialéctico permite entenderla como un sistema estructurado por antagonismos y luchas.

Is a dialectical system in which cultural action and cultural structures go online: It involves the permanent mutualproduction of practices and structures that produce and re-create mind (ideas, values, affects, meaning, taste) and body with the help of networked computer technology that allows to transcend spatial borders and takes place synchronously or asynchronously. Cyberculture develops dynamically; it is a self-organizing system in which cultural practices and structures permanently produce and reproduce each other in self-referential loops. Such a dialectical notion of cyberculture avoids the onesidedness of subjective and objective approaches. (Fuchs, 2008, p. 299)

Lo que caracteriza a la cibercultura es que su origen se basa en la tecnología; esto segmenta su producción a la dependencia de dispositivos técnicos y marca unos rasgos propios en diversas prácticas sociales alrededor de dichos dispositivos, conectados la mayoría de las veces a las redes de computación o teléfonos celulares. Con ello se abre un abanico de miradas sobre nuevas formas de percibir, apreciar y vivir el mundo que habitamos. ¡Aunque no todo son bondades! El uso irresponsable del término cibercultura nos lleva a una vacuidad de su sentido en tanto puede no atender las implicaciones políticas e ideológicas de los procesos de los grupos 
sociales y dejarían de tenerse en cuenta las relaciones de poder que median sobre las condiciones de acceso de los grupos a las tecnologías. Moya y Vazquez (2010) haciendo alusión a García Canclini, plantean que no podemos omitir tres aspectos condicionantes de las posibilidades de participar o de formar parte de la cibercultura 1) La accesibilidad al medio "internet”; 2) La apropiación de este medio; 3) Las capacidades simbólicas necesarias para su operación.

\title{
Las transformaciones en el consumo de la telefonía celular en Latinoamérica
}

\author{
Estamos ante una nueva tecnicidad, en la que la técnica ha trascendido su \\ habitual papel de mero instrumento hacia un nuevo estatus: el de dimensión \\ estructural de las sociedades contemporáneas
}

(Martín Barbero 2006).

Pareciera que la tecnología es hoy eje vertebral de la cultura. En la medida en que conforma la cotidianidad de las personas, la tecnología está dando lugar a transformaciones sociales de grandes proporciones; por esta razón podríamos afirmar que no tiene sentido sostener una visión simplista y puramente instrumental de ella ${ }^{9}$.

Latinoamérica no es ajena a los grandes cambios en las dinámicas del mundo y presa de la globalización también experimenta durante las primeras décadas del siglo XXI un crecimiento en el número de celulares y conexiones a internet entre sus habitantes.

“A 2008 la base de suscriptores superaba los 300 millones en tecnología GSM y 69,2 millones en plataforma CDMA, con proyecciones de crecimiento por encima de los 500 millones de suscriptores para el año 2012, con un promedio superior a las 80 líneas por cada 100 habitantes; Argentina, Uruguay, Venezuela, Chile, Ecuador y Colombia son los países con el registro de mayor cobertura en la región" (Ontiveros Baeza et al. 2009)

Los datos anteriores pueden ser vistos como el número de artefactos que circulaban por las calles de América latina y cifras que rápidamente dejarán de servir porque la producción de equipos no da espera, pero más allá de eso ponen en evidencia un nuevo horizonte de tramas en los modos como se comunican las personas y las implicaciones en términos culturales y por ende de consumo que este fenómeno supone.

En este escenario las personas experimentan cambios en la forma como perciben el tiempo, el espacio, la diversión, y las diferentes formas de relacionarse entre ellas y su entorno que desdibujan las reglas y patrones estructurales que reinaban antes. Los celulares e Internet han sido el vehículo en el que las sociedades se han 
trasladado del entorno físico, en el que siempre las concebimos, a un entorno virtual en el que las dinámicas y hábitos sociales y de comportamiento de los individuos se han visto afectados. "Hoy vemos nacer y crecer a una generación de jóvenes que intenta identificarse a través nuevas estrategias de comunicación, y de nuevos usos y apropiaciones de tecnologías de comunicación” (Treviño \& Millán, 2007, p. 16) Es la identidad que se gesta en el movimiento des-territorializador que atraviesan las demarcaciones culturales pues, desarraigadas, las culturas tienden inevitablemente a hibridarse, como lo indica Martín Barbero (2002).

Sin embargo, es necesario volver en este punto a lo propuesto anteriormente sobre las condiciones de acceso a la tecnología. Las nuevas generaciones de celulares y las ventajas que con ellas llegan a los usuarios no son de acceso público, la economía implanta una brecha digital en la que no todos pueden obtener los últimos avances, por eso las conclusiones totalizadoras sobre estas bondades instrumentales son nocivas para dejar de manifiesto la desigualdad que no sólo se presenta en este ámbito, sino que permea todas las esferas sociales. La brecha digital puede ser un factor de exclusión social o indicador de estatus de acuerdo a las posibilidades de adquisición de un teléfono celular y en relación al tipo de celular que se tenga (Treviño y Millán, 2007).

El teléfono inteligente también ha transformado las formas de escritura, en un principio con mensajes de texto de 160 caracteres y hoy día con las aplicaciones de mensajería instantánea que llegan a suprimir el fin con el que fue concebido este instrumento: recibir y hacer llamadas de voz. Según Salinas \& Thompson (2011) algunos teóricos valoran esta nueva forma de "conversación” debido a la simplicidad, la simbología y el uso de imágenes que sacan la escritura de la rigidez de las reglas convencionales y estimulan la comunicación.

\section{Georeferenciando a Colombia}

Colombia no es ajena a las transformaciones descritas para los países de América latina. El final de la primera década del nuevo siglo situó al país como el de mayor crecimiento de América Latina en cuanto a penetración de Internet. A finales de 2009, Colombia registró un incremento del 32\% frente al 23\% consolidado de la región (Fosk, 2010).

Una realidad muy diferente a la registrada durante los primeros años de la nueva década, en la que el país se situó a la saga en cuanto a la implementación de nuevas tecnologías de comunicación. Sin embargo, la política oficial de apoyo al sector, sumada a un clima favorable para la inversión extranjera atraída por un mercado potencial muy grande, han sido factores que le permitieron avanzar al país en materia de telecomunicaciones en los principales centros urbanos (Arango Forero, G., Bringué Sala, X., \& Sádaba Chalezquer, C. 2010, p.147). 
En años más recientes se habla de un estado de madurez digital para el país, cuando estudios como el de Google Consumer Barometer (2017) presentan un punto de saturación para el uso del internet en el país, principalmente por personas mayores de 16 años.

En relación con el uso del celular, punto central de análisis de este documento, desde el 2010 ya se evidenciaba un panorama bastante interesante, para el caso específico de los millennials colombianos. Arango Forero et. al. (2010) explican cómo para el inicio de la segunda década de este milenio el Smarthphone representaba para los adolescentes un elemento necesario para la organización del tiempo, gracias a las funciones de reloj y de agenda, además de ser un elemento de suma importancia en las dinámicas de relacionamiento, desarrollo de habilidades sociales y de interacción.

Para el año 2016, el estudio realizado por IMS y ComScore (2016) indica para Colombia un incremento en el uso del teléfono móvil, no solo para el grupo de los millennials que pasaba hasta 120 minutos al día interactuando con estos elementos, sino también en usuarios de otras generaciones que reportaron hasta 100 minutos/ día de interacción.

Los cambios en las prácticas y modos de uso del celular han significado la reconfiguración del estilo de vida en el país. Es imposible que se produzcan cambios a este nivel del comportamiento colectivo sin que supongan grandes transformaciones culturales a partir del uso de esta pantalla. Un incremento en la cobertura del internet móvil y el posicionamiento del celular como un artículo de primera necesidad le abrieron paso a un instrumento del que hoy es muy difícil separarse. Muestra de ello es que en el país se encuentren más hogares con celular que con acueducto. "Mientras que en el 96,5 por ciento de los hogares colombianos hay al menos un teléfono celular, solo el 89,5 por ciento de estos cuenta con acceso a uno de los servicios básicos fundamentales: el agua”. (DANE, 2016b)

Pareciera que para los usuarios cada vez es más difícil desarrollar actividades sin la compañía de estos dispositivos: entretenimiento, información, búsquedas, compras online, transporte, pago de servicios, deporte o trabajo pueden ser potenciados a través del apoyo que brindan estos aparatos. Según, el estudio de consumo móvil Deloitte 2018, la mayoría de los usuarios de Smarthphone en Colombia han empezado a utilizar su celular para realizar algún tipo de acción financiera o comercial, incluyendo opciones de navegación y consulta” (Deloitte, 2018, p. 34) Por esta razón, seguramente se incrementará en los próximos años la oferta de entidades financieras y comercios, así como la confianza en las aplicaciones móviles para realizar acciones que impliquen movimiento de dinero. Este aumento en el número de aplicaciones descargadas, sumado a la variedad de actividades realizables, indica un deseo de hallar la mejor aplicación para cada tipo de necesidad, más que una aplicación única que intente resolverlo todo. 


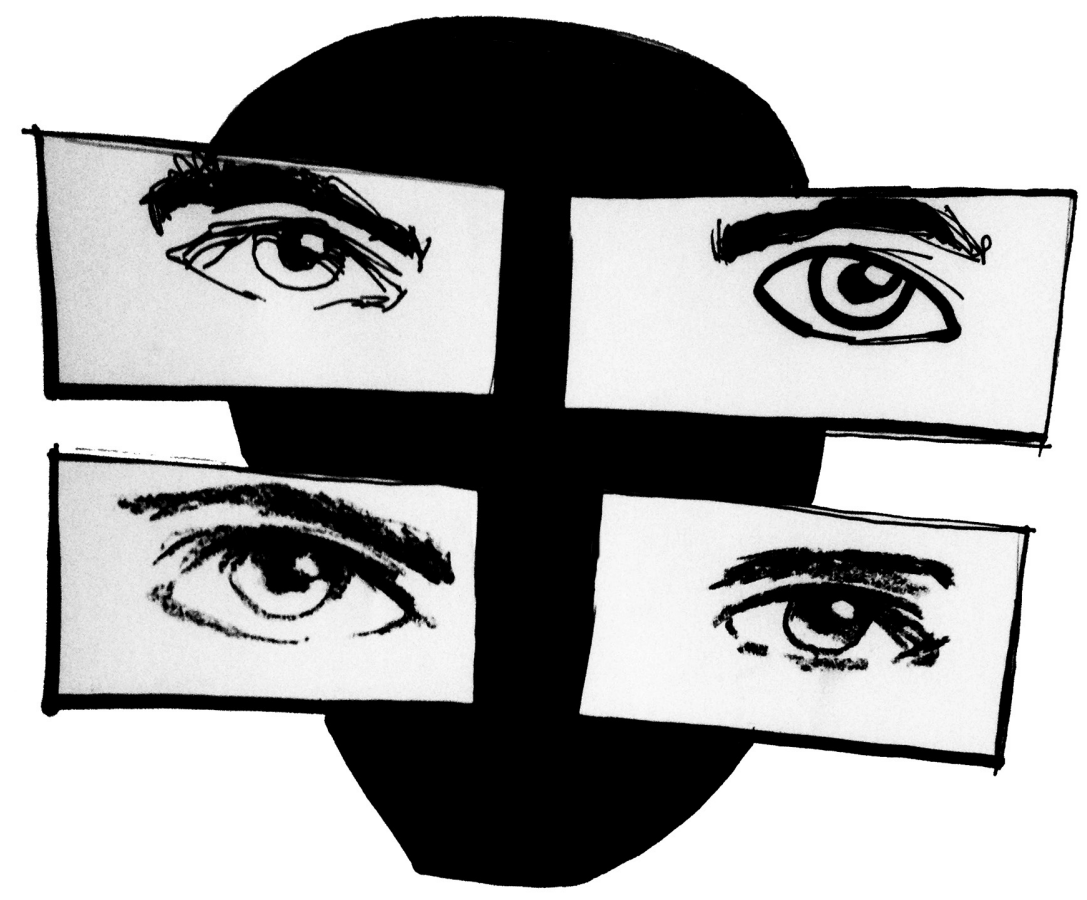

Existen otros elementos que llaman la atención en los estudios más recientes de consumo móvil en el país (Deloitte, 2018). Por ejemplo, la inclusión de la pregunta sobre las formas en cómo los usuarios auto-limitan el uso del teléfono inteligente. Encontrando entre las principales estrategias, guardar el dispositivo en un bolso o un bolsillo cuando se reúne con otros, apagar el aparato o eliminar ciertas aplicaciones. También se observa para Colombia un abaratamiento de los planes de datos en los últimos años, lo que garantiza un mayor de número de personas conectadas en todo momento; sin embargo, parece que las ofertas comerciales de los operadores móviles no terminan de satisfacer los deseos de los usuarios, pues tan sólo 1 de cada 3 colombianos usuarios del servicio de Internet Móvil se siente satisfecho con la tarifa que paga (Deloitte, 2018).

Cada una de los estudios citados esboza un panorama sobre cómo las industrias culturales captan en la actualidad a sus audiencias y de qué manera estas últimas interactúan constantemente con tecnologías como el teléfono móvil, para crear nuevos significados y diferentes interacciones virtuales y físicas que permiten interpretar un mundo que ya no es sólo análogo, ni se puede definir sólo en términos de realidades espacio-temporales. En este sentido, también es necesario reflexionar sobre la manera individual o personalizada en la que se han transformado estos consumos. Las funciones bastante estructuradas y limitadas de los primeros teléfonos, contrastan con la actual capacidad de agencia de los usuarios (Latour, 2008) que se reflejan principalmente en la selección de aplicaciones y consumo de contenidos a la carta. 
La introducción de la tecnología en la vida cotidiana trae aparejadas la emergencia de nuevos espacios, nuevas formas de participación y socialización, nuevas maneras de relacionarse y de pensar el mundo en el cual vivimos. Es por esta razón que es necesario pensarse continuamente las transformaciones en los estilos de vida que genera la telefonía celular con el uso de los Smartphones. La irrupción de esta cuarta pantalla a la vida cotidiana de los colombianos trae consigo no sólo un electrodoméstico más que se agrega a la lista de soluciones que la tecnología brindaba para el hogar, sino un artefacto que permeó la cotidianidad y se convirtió casi en una extensión de la existencia, así esta tenga lugar en el plano de la virtualidad (que cada vez es más difuso). La cibercultura que se genera desde el uso de estos dispositivos no es buena ni mala, puede tener elementos cooperativos o competitivos; de ahí la posibilidad de entender las dinámicas propias de las condiciones contradictorias de las sociedades modernas (Salinas \& Thompson, 2011).

Es tal el alcance de la irrupción del celular en la vida de los colombianos que lo utilizan hasta para acompañar sus idas al baño o no se separan de él mientras comparten con amigos en un bar, es un elemento que genera un puente en doble vía entre lo público y lo privado. Los usuarios también han entrado en dinámicas exhibicionistas potenciadas por la interacción de los mismos en las redes sociales. Estas se convierten en escenario ideal para se realicen transmisiones en simultáneo de la vida privada, que dan cuenta de sus recorridos, de las personas con las que regocijan su alma, de los sentimientos que los afectan o alegran y en un sentido teórico más abstracto de sus consumos culturales, académicos o de entretenimiento; todo esto a través del cristal del celular que se ha transformado en una ventana para mostrarse al mundo.

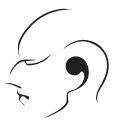




\section{Notas}

${ }^{1}$ Comunicadora Social de la Universidad del Valle (Cali-Colombia), especialista en Comunicación Organizacional de la Universidad Autónoma de Occidente (Cali-Colombia) y Magister en Comunicación de la Universidad del Norte (Barranquilla-Colombia) Estudiante doctorado en Sociología, Universidad del Valle. Actualmente se desempeña como docente catedrática de la Escuela de Comunicación social de la Universidad del Valle.

2 Comunicador Social y Magister en Historia de la Universidad del Valle (Cali- Colombia). Actualmente, se desempeña como docente catedrático de la licenciatura en historia de la Universidad del Valle y como docente investigador de tiempo completo de la Facultad de Comunicación y Publicidad, Universidad Santiago de Cali.

${ }^{3}$ La obsolescencia programada es la determinación o programación del fin de la vida útil de un producto, de modo que, tras un período de tiempo calculado de antemano por el fabricante durante la fase de diseño de dicho producto, este se torne obsoleto, no funcional, inútil o inservible. Video: La historia de las cosas. https://www.youtube.com/watch?v=lrz8FH4PQPU. Consultado: 29 de septiembre de 2018.

${ }^{4}$ Hombre-medio: un ser ficticio para quien todas las cosas ocurrirán conforme a los resultados medios obtenidos por la sociedad. (Quetelet, 1835)

${ }^{5}$ Este nuevo momento del internet ha sido vendido como una gran revolución. "Es una de las tendencias más manidas en los discursos ejecutivos y una de las que se incluyen en todos los planes de negocio y presentaciones al mercado, [ en donde] miles de millones de objetos conectados van a darnos más información de la que jamás habríamos imaginado y un nivel de automatización nunca antes visto. Pero esta inminente realidad parece que está teniendo más problemas de los deseados para despegar" (Ticbeat, 2017) Para el caso específico de Colombia la situación es similar a la descrita para el resto del mundo. El internet de las cosas es una tendencia inmadura, mientras que el uso del teléfono inteligente "se sigue masificado en poco tiempo y de manera similar en todas las edades" (Deloitte, 2018).

${ }^{6}$ En este acto de garantizar la seguridad del perfil del usuario se observan varias de las características de la cibercultura descritas por Cuadra (2003): A. Cada usuario deja de ser identificable por referencia a un estrato social. B. La 'cibercultura' ha elevado a la enésima potencia la capacidad colectiva de representar realidades miméticas y abstractas. C. La 'cibercultura' constituye, según hemos visto, un nivel de complejidad semiótica, inédita en la historia humana. (Cuadra, 2003, pps. 66-67)

${ }^{7}$ En Colombia, dada la existencia de regulación que controla la recolección y el uso de la información personal, las compañías financieras y comercios deberán adelantar acciones para obtener consentimiento expreso para el uso de la información personal que requieren a fin de darle una mejor experiencia al usuario. (Deloitte, 2018)

8 Según la encuesta Deloitte 2018, sobre consumidores móviles, en Colombia.

${ }_{9}$ Esta construcción tecnológica de la cotidianeidad es lo que Piscitelli llama tecno-culturas. (Piscitelli, 2002)
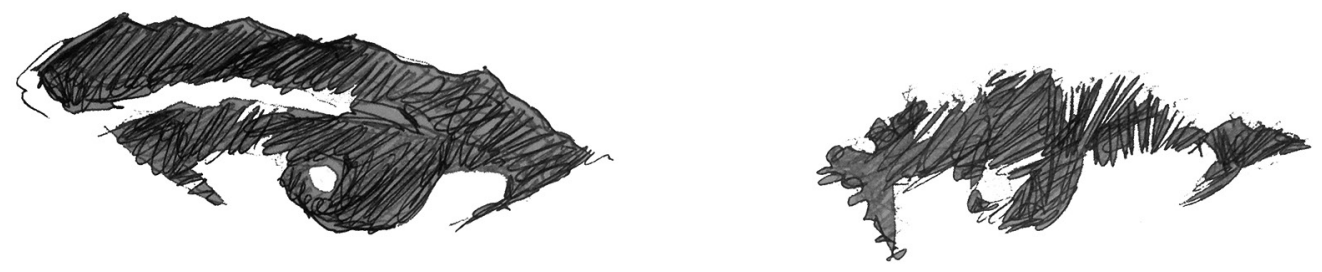


\section{Referencias bibliográficas:}

Arango Forero, G., Bringué Sala, X., \&Sádaba Chalezquer, C. (2010). La generación interactiva en Colombia: adolescentes frente a la Internet, el celular y los videojuegos. Anagramas-Rumbos y sentidos de la comunicación-, 9(17), 45-56.

Ahonen, T. (2008). Mobile as 7th of the mass media: Cellphone, cameraphone, iphone, smarthphone. FuturetexT. Londres.

Castells, M. et al. (2007). Comunicación móvil y sociedad, una perspectiva global. Edición electrónica gratuita. Recuperado en: www.eumed. net/libros/2007c/312/. Consultado: 24 de septiembre de 2018.

(2006). La sociedad red: una visión global. Alianza Editorial. Madrid, España.

Costa-Sanchez (2014). Las singularidades del medio móvil: integración multimedia, personalización, geolocalización y participación. Estudio de su presencia en las Apps de la prensa española. Palabra Clave 17 (3), 672-694. DOI: 10.5294/pacla.2014.17.3.5

Cuadra, A. (2003). De la Ciudad Letrada a la CiudadVirtual. LOM. Ediciones, Santiago de Chile, Chile

DANE (2016a). Encuesta Nacional de Consumo Cultural. [Documento www] https: / / formularios.dane. gov.co/Anda_4_1/index.php/catalog/447/export Consultado: 3 agosto de 2018.

DANE (2016b). Encuesta Nacional de Calidad de Vida. [Documento www] https://www.dane.gov. co/index.php/estadisticas-por-tema/salud/calidad-de-vida-ecv/encuesta-nacional-decalidad-de-vida-ecv-2016. Consultado: 30 junio de 2018.

DELOITTE (2018). Encuesta Global de Consumidores Móviles 2018 - Edición Colombia. [Documento www] https://www2.deloitte.com/co/es/pages/technology-media-and-telecommunications/ articles/consumo-movil-2018.html Consultado: 30 enero de 2019.

Encuesta Global de Consumidores Móviles 2015 - Edición Colombia. La distracción de los móviles. [Documento www] http://www2.deloitte.com/co/es/pages/technologymedia-and-telecommunications / articles/ encuesta-global-de-consumidores-moviles--2015---deloitte-colomb.html. Consultado: 28 septiembre de 2016.

Evans, D. (2011). Internet de las cosas. Cómo la próxima evolución de internet lo cambia todo. Cisco Internet Business Solutions Group (IBSG). [Documento WWW] http://www.cisco.com/c/dam/ global/es_mx/solutions/executive/assets/pdf/internet-of-things-iot-ibsg.pdf Consultado: 28 septiembre de 2016.

Fosk, A. (2010). Situación de internet en Latinoamérica. [Documento www] http://www.slideshare. net/ Consultado: 29 de septiembre de 2018

Fumero, A. (abril- junio, 2010). La red en el móvil. Telos, cuadernos de comunicación e innovación. Movilidad y nuevas fronteras. No. 83. España.

Fuchs, C. (2008). Internet and Society: Social Theory in the Information Age. Routledge. Routledge Research in Information Technology and Society Series. Number 8. New York.

García López, X.; Westlund, O.; \& Silva Rodríguez, A. (2015). Prensa, smartphones y tablets. La industria de medios impresos se sube al periodismo móvil. Telos: Cuadernos de comunicación e innovación (100), 128-139.

García, K. (2014). Gramática de los nuevos medios y mutaciones tecnoculturales. Esbozos para un mapa de la cuestión. En: Nexus, Comunicación. No.15. Universidad del Valle, Cali. Colombia.

Gelmato (2019). Red 5G. Características y usos de esta tecnología. [Documento www] https://www. gemalto.com/latam/telecom/inspiracion/5g Consultado: 29 de marzo de 2019

Google ConsumerBarometer (2017). Estudio de ConsumerBarometer. [Documento www] www. consumerbarometer.com Consultado: 29 de septiembre de 2018

IMS \& COMSCORE (SEPTIEMBRE, 2016). Mobil in latam study. [Documento www] https: / / www. imscorporate.com/news/Estudios-comScore/IMS-Mobile-Study-Septiembre2016.pdf Consultado: 8 de julio de 2018.

Latour, B. (2008). Reensamblar lo social: una introducción a la teoría del actor-red. Manantial. 1era edición. Buenos Aires, Argentina. 
Martín Barbero, J. (2002). Jóvenes: Comunicación e identidad. En Pensar Iberoamerica Revista de Cultura Número 0, febrero 2002.

Mattelart, A. (2007). Historia de la sociedad de la información. Paidós. Barcelona, España.

Mattelart, A. \& Mattelart, M. (2001). Historia de las teorías de la comunicación. PaidosComunicación. Barcelona, España.

Mazlish, B. (1993). The fourth discontinuity. The co-evolution of men and machines. New Heaven, ct: Yale University Press.

Mokyr, J. (1990). The lever of Riches: Technological creativity and economic progress. Oxford: Oxford UniversityPress.

Moya, M., \& Vázquez, J. (2010). De la Cultura a la Cibercultura: la mediatización tecnológica en la construcción de conocimiento y en las nuevas formas de sociabilidad. Cuadernos de antropología social, (31), 75-96.

Ontiveros Baeza, E., Martín Enríquez, A., Fernández de Lis, S., Rodríguez Teubal, I. \& López Sabater, V. (2009). Telefonía móvil y desarrollo financiero en América Latina. Barcelona: Ariel.

Piscitelli, A (2002). MetaCultura: El eclipse de los medios masivos en la era del Internet. La Crujía ediciones. Buenos Aires.

Quetelet, A. (1999). Física social o Ensayo sobre el hombre y el desarrollo de sus facultades. Reis: Revista española de investigaciones sociológicas (87), pps. 305 - 324.

Salinas, N. \& Thompson, C. (2011). La cibercultura desde una sociología de internet. Revista F@ro. Revista teórica de la Facultad de Ciencias Sociales. e-ISSN 0718-4018 Monográfico Año 7 Número 13 - I Semestre Universidad de Playa Ancha. Valparaíso, Chile.

Sánchez, U., Botero, L. \& Giraldo, A. (2012). Modelos de comunicación digital. Universidad de Medellín. Colombia.

Treviño, E. F., \& Millán, O. A. (2007). La influencia de la telefonía celular en el entorno social de los jóvenes universitarios en Tampico Tamaulipas (México). Trabajo presentado en el Foro Internacional sobre Comunicación e Xuventude. Galicia, España.

TICBEAT (2017). El Internet de las Cosas sigue sin despegar. [Documento www] https: / / www.ticbeat. com/innovacion/el-internet-de-las-cosas-sigue-sin-despegar-apenas-supone-el-10-de-lospresupuestos-tic/ Consultado: 30 de enero de 2019.

Recibido: 22 de Abril del 2019 / Aprobado: 30 de Mayo de 2019 